\title{
ENFERMAGEM NA WEB: O PROCESSO de CRIAÇÃO E VALIDAÇÃo de UM WEB SITE SOBRE DOENĢA ARTERIAL CORONARIANA ${ }^{1}$
}

Isaac Rosa Marques ${ }^{2}$

Heimar de Fátima Marin ${ }^{3}$

Marques IR, Marin HF. Enfermagem na web: o processo de criação e validação de um web site sobre doença arterial coronariana. Rev Latino-am Enfermagem 2002 maio-junho; 10(3):298-307.

A World Wide Web é uma importante fonte à pesquisa da informação de saúde. Um desafio para a área de Informática em Enfermagem brasileira é o de usar esse potencial para promover a educação em saúde. Este artigo objetiva apresentar um modelo de criação e validação usado em um Web site educativo, denominado CardioSite, cujo tema é a Doença Arterial Coronariana. Para a criação do mesmo, foi adotado um método com fases de modelagem conceitual, de desenvolvimento, de implementação e de avaliação. Na fase de avaliação, a validação foi feita através de um painel on-line com especialistas nas áreas de informática e saúde. Os resultados demonstraram que as informações são confiáveis e válidas. Considerando a inexistência de sistemas nacionais oficiais com esse propósito, este modelo demonstrou-se efetivo para avaliar a qualidade do conteúdo do Web site.

DESCRITORES: internet, enfermagem, coronariopatia

\section{NURSING ON THE WEB: THE CREATION AND VALIDATION PROCESS OF A CORONARY HEART DISEASE WEB SITE}

The World Wide Web is an important health information research source. A challenge for the Brazilian Nursing Informatics area is to use its potential to promote health education. This paper aims to present a developing and validating model used in an educational Web site, named CardioSite, which subject is Coronary Heart Disease. In its creation it was adopted a method with phases of conceptual modeling, development, implementation, and evaluation. In the evaluation phase, the validation was performed through an online informatics and health experts panel. The results demonstrated that information was reliable and valid. Considering that national official systems are not available to that approach, this model demonstrated effectiveness in assessing the quality of the Web site content.

DESCRIPTORS: internet, nursing, coronary disease

\footnotetext{
${ }^{1}$ Resultados da Dissertação de Mestrado em Enfermagem da Universidade Federal de São Paulo (UNIFESP), aprovada pelo Comitê de Ética em Pesquisa do Hospital São Paulo, Ref. CEP № 414/00; ${ }^{2}$ Aluno do Curso de Doutorado em Enfermagem, Professor Adjunto do Curso de Enfermagem do Centro Universitário Adventista de São Paulo (UNASP), Universidade Santo Amaro (UNISA), e-mail: isaacmarx@hotmail.com; ${ }^{3}$ Doutor em Enfermagem, Professor Adjunto do Departamento de Enfermagem, Orientadora do trabalho. Universidade Federal de São Paulo
} 


\section{ENFERMERÍA EN LA WEB: EL PROCESO DE CONSTRUCCIÓN Y VALIDEZ DE UNA PAGINA WEB SOBRE LA ENFERMEDAD ARTERIAL CORONARIA}

La Word Wide Web es una importante fuente de investigación de información sobre la salud. Un desafío para el área de Informática en Enfermería brasileña es el de usar este potencial para promover la educación en salud. Este artículo busca presentar un modelo de creación y evaluación utilizado en una Pagina Web educativa, denominado CardioSite, cuyo tema es la Enfermedad Arterial Coronaria. En la creación del mismo se ha adoptado un método con las fases de modelaje conceptual, de desarrollo, de implementación y de evaluación. En la fase de evaluación, la validación se llevó a cabo por medio de un panel on-line con especialistas en las áreas de informática y salud. Los resultados señalaron tales informaciones como confiables y validas. Al considerar la inexistencia de sistemas nacionales oficiales con este propósito, el modelo se ha mostrado efectivo para evaluar la calidad del contenido la pagina web.

DESCRIPTORES: internet, enfermería, coronariopatía

\section{INTRODUÇÃO}

$\boldsymbol{O}$ sistema Internet revolucionou o modo de usar o computador e as formas de comunicação, sem qualquer precedente de comparação. A Internet é um sistema físico de arquitetura aberta capaz de interligar computadores, utilizando um padrão de protocolos para a troca de informações. Para interligar os computadores é necessário um sistema de gerenciamento para a transferência e controle das informações comuns à toda a rede. Esse sistema é formado por Protocolos de Controle de Transmissão e Protocolo Internet [Transmission Control Protocol e Internet Protocol - TCP/IP], os quais garantem a integridade dos dados que trafegam no meio e uniformizam a comunicação entre diferentes redes $^{(1-2)}$.

Os protocolos TCP/IP estão embutidos em um dispositivo de hardware chamado de modulador/emulador [modem]. Para acessar o sistema Internet, são necessários um computador equipado com modem, uma linha telefônica, um software [browser] capaz de interpretar as mídias recebidas por meio dos impulsos telefônicos, transformando-as em objetos visuais, e a contratação dos serviços de um provedor de acesso à Internet ${ }^{(1)}$.

Ao contrário do que se pensa, a Internet não é um produto da tecnologia atual. Sua história teve início por volta de 1960, com a construção da rede de informações do exército americano, por ocasião da Guerra Fria. A ARPA [Advanced Research Projects Agency], uma agência de pesquisas e desenvolvimento tecnológico do Departamento de Defesa dos Estados Unidos da América, foi encarregada de construir uma rede de longa distância capaz de interligar computadores espalhados naquele país para o tráfego de dados exclusivos da área militar. No início da década seguinte, a Internet passou a ser utilizada por algumas universidades, sobretudo para o desenvolvimento de pesquisas. Por essa época, foram criados os serviços de transferência de arquivos, mais tarde chamados de Protocolos de Transferência de Arquivos [FTP - File Transfer Protocol]. A criação desses protocolos deu origem ao serviço do correio eletrônico, o serviço mais usado no sistema Internet. Assim que a tecnologia foi evoluindo, surgiu a World Wide Web, no final da década de 1980, na Suíça. Esse foi o grande passo para a popularização da Internet ${ }^{(1-2)}$. 
A WWW ou Web, como é mais conhecida, é o conjunto de documentos baseados na linguagem de hipertexto, existentes nos milhares de computadores ao redor do mundo e que podem ser acessados através do sistema Internet. Mesmo que o intuito do criador da WWW fosse apenas o de disponibilizar documentos científicos de uma forma simplificada e acessível, sua utilização tornou-se bastante atrativa para outras finalidades, incluindo-se, em primeira instância, os interesses comerciais. À medida que a utilização desse sistema alcançou grande popularidade para a disponibilização de informações, outras áreas das atividades humanas fizeram-se presentes na rede mundial de computadores ${ }^{(1)}$.

Com a popularização da Internet, novos termos passaram a ser inseridos em nosso vocabulário. Exemplos de alguns deles são: página Web, que significa um documento apresentado sob o formato de HTML [linguagem marcada de hipertexto]; Web site, que é um conjunto de páginas Web organizadas ou abrigadas sob um determinado domínio [URL - Uniform Resource Locator]. Outro termo é Web designer, o qual se refere ao profissional que desenvolve Web sites.

De uma forma progressivamente crescente, a Internet vem se fazendo presente no cotidiano de parte da população. Isso se deve à liberação da Internet para fins comerciais, a partir do início da década de 1990 e da expansão comercial da venda de computadores pessoais, o que a tornou mais acessível à população. Ao perceber a Internet como um novo canal de disseminação de informação, inúmeras empresas vêm se fazendo presentes na rede, por meio de seus Web sites, criando facilidades e novas formas de expansão para os seus negócios.
Diante dessa evolução, a enfermagem, a exemplo de outras profissões da área de saúde, também começou a perceber a Internet como um meio através do qual a informação de saúde poderia ser mais bem disseminada, seja ela para o intercâmbio profissional, como recurso para a pesquisa e/ou como uma ferramenta para promover a educação em saúde.

Internet e Enfermagem

Usar a Internet para promover a educação em saúde é um desafio para a área de Informática em Enfermagem no Brasil ${ }^{(3)}$. $\mathrm{O}$ poder da Internet e seu impacto em questões que envolvem os aspectos do dia a dia da vida humana é algo que não pode ser desprezado nos dias atuais. Como essa tecnologia tem sido pouco explorada pela enfermagem, é necessário estar alerta às mudanças que já são presentes e para as que virão no futuro. Uma dessas mudanças poderá ser o surgimento de novos produtos e mercados nos quais a enfermagem poderá inserir-se.

Como um recurso global que conecta milhões de computadores, a Internet é uma excelente plataforma na qual a enfermagem pode buscar o seu desenvolvimento e aperfeiçoamento como profissão. As projeções a curto prazo estimam que esse recurso atuará como a infra-estrutura central em saúde, ajudando enfermeiros a estenderem seus serviços à comunidade e a educarem populações específicas ${ }^{(4)}$. Tomando como base a crescente tendência do uso da tecnologia da informação no cotidiano da enfermagem, é importante considerar a sua finalidade específica em colaborar para que o processo de atenção à saúde resulte em benefícios, tanto para os usuários, como para os profissionais 
que prestam a assistência ${ }^{(5)}$. A presença da enfermagem na promoção de educação em saúde via Internet está cada vez mais se consolidando como uma prática comum. A literatura fornece abundantes relatos de tais utilizações que visam ao suporte em nível informativo, tanto para a população ${ }^{(6-8)}$ como para o próprio profissional $^{(9)}$.

Ao perceber o potencial da Internet para a disseminação da informação de saúde, surgiu a idéia de construir um Web site com a finalidade específica de promover a educação em saúde. O tema escolhido foi a Doença Arterial Coronariana [DAC], levando em conta o aumento na incidência dessa doença nos últimos anos, segundo dados estatísticos do Sistema de Informações sobre Mortalidade do Ministério da Saúde ${ }^{(10)}$. Os objetivos do estudo foram: descrever um modelo de desenvolvimento de um Web site com informação de saúde e validar a qualidade da informação produzida.

\section{MATERIAL E MÉTODO}

Este trabalho constituiu-se numa pesquisa experimental descritiva inserida na Área de Concentração de Saúde do Adulto, Linha de Pesquisa de Informática em Saúde, Eixo Temático - Sistemas Educacionais, do Programa de Pós-Graduação - Curso de Mestrado, do Departamento de Enfermagem da Universidade Federal de São Paulo [UNIFESP]. A pesquisa baseou-se na construção ativa de um Web site, sendo o seu referencial teórico o modelo de desenvolvimento de Web sites com fases de modelagem conceitual, de desenvolvimento, de implementação e de avaliação ${ }^{(11)}$.
Fase de modelagem conceitual

A primeira fase foi a de modelagem conceitual. Nessa fase, buscou-se selecionar os conteúdos que constituiriam a informação a ser disponibilizada no Web site. As fontes primárias de informação constituíram-se de livros-texto, artigos de periódicos e informações publicadas na Web, desde que oriundas de fontes confiáveis. Os Web sites institucionais da American Heart Association [Associação Americana do Coração - http:// www.americanheart.org] e da InteliHealth [http:/ /www.intelihealth.com] serviram como modelos para a organização do conteúdo da informação. Para atribuir qualidade à informação, foram selecionados os Códigos de Conduta para Web sites Médicos e de Informação de Saúde da Health on the Net Foundation - $\mathrm{HON}^{(12)}$ e as diretrizes do Instituto Tecnológico da Informação de Saúde [Health Information Technology Institute $-H I T]^{(13)}$.

Fases de desenvolvimento e implementação

A segunda fase foi a de desenvolvimento. Antes da construção das páginas Web, as mídias [texto, imagens e animações] que fizeram parte do conteúdo, foram previamente preparadas com o uso de editor de texto, digitalizador [scanner] e editor de imagens. Para a construção das páginas Web, foi utilizado como plataforma um software de desenvolvimento visual [FrontPage 3.0 da Microsotf Corporation $\mathbb{B}$ ], evitando-se maior gasto de tempo com a escrita integral por códigos na linguagem HTML.

A terceira fase foi a de implementação. Nessa fase, o conteúdo do Web site foi hospedado em um servidor comercial na 
seguinte URL: http://www.info.saude.nom.br/ cardiosite/. Essa opção foi escolhida devido à flexibilidade para atualização do material, prevendo encontrar essa dificuldade, caso a publicação/hospedagem fosse efetuada no servidor Web institucional.

Fase de avaliação

A última fase foi a de avaliação. Dois métodos podem ser usados para avaliar um Web site: a) por meio de pesquisa de opinião com usuários, para saber se os objetivos do material estão sendo alcançados e b) por meio da validação por especialistas antes de o material ser colocado à disposição do usuárioalvo. Neste trabalho, um painel on-line foi composto por profissionais das áreas de saúde e informática. Para encontrar profissionais dessas áreas que pudessem compor os grupos de consultores, uma pesquisa na Web foi procedida. Para tanto, os mecanismos de busca como: Altavista [http:www.altavista.com], Cadê [http://www.cade.com.br] e Achei [http:// www.achei.com.br] foram acessados, usandose os unitermos: profissionais de cardiologia, hospitais de cardiologia, enfermagem em cardiologia e desenvolvedores Web. Após a obtenção do endereço de correio eletrônico de possíveis consultores, 92 mensagens eletrônicas foram enviadas. Ao obter contato com os mesmos, o próximo passo foi seguir os critérios de inclusão dos consultores. Para a área de informática [Grupo I], o critério de inclusão era ter experiência na atividade de Web designer por tempo maior que cinco anos. Para o Grupo II, formado por enfermeiros e médicos, os critérios de inclusão foram os seguintes: estar vinculado à uma instituição especializada em cardiologia, ter experiência profissional na área de cardiologia há pelo menos cinco anos, ter título de especialista nessa área e ter acesso à Internet. Cada consultor participante do estudo assinou o Termo de Consentimento Livre e Esclarecido, enviado via correio convencional e devolvido ao condutor do estudo. Após a formação dos grupos, cada consultor teve o prazo de 30 dias para avaliar o Web site. Em seguida, os mesmos validaram o material através de um questionário eletrônico do tipo escala Likert, adaptado de modelo publicado na Web ${ }^{(14)}$. Para os consultores do Grupo I, os critérios de validação estão apresentados na Tabela $1 \mathrm{e}$, para o Grupo II, os apresentados na Tabela 2.

Tabela 1 - Critérios e itens do instrumento de validação aplicado aos consultores do Grupo I

CRITÉRIOS ITENS

1. Autoridade

A. Existe clara indicaçẫo sobre o autor do Web sitee sua qualificaçẫo. B. Existemmecanismos pelos quais é possivel estabelecer contato com o autor.

2. Velocidade A. A velocidade de carregamento da páginainicial.

B. Avelocidade de carregamento das demais páginas.

3. Design

A. A página inicial apresenta um desenho atrativo que induz o usuário a navegar pelo Web site.

B. A página inicial apresenta um desenhoclaroe suficientemente capaz de ser manipulado com sucesso por usuários comuns.

4. Navegabilidade A. O usuário tem facilidade de navegaçẫo página a página, seçẫo a seçẫo, ou de um link para outro, sem ficar perdido.

B. Todos os links estẫo claramente definidos e servem a um propósito facilmente identificado.

C. Os links colocados à disposiçẫo para outras páginas ou Web sites extemos operam eficientemente.

5. Uso de gráficos A. OS gráficos ou imagens estẫo claramente apresentados.

B. Os gráficos ou imagens servem a um propósito claro e apropriado para os usuáriosa que se destinam. 
Tabela 2 - Critérios e itens do instrumento de validação aplicado aos consultores do Grupo II

\section{CRITÉRIOS ITENS}

1. Autoridade

A. Existe indicação clara sobre o autor do Web site e sua qualificação.

B. Existem mecanismos pelos quais é possível estabelecer contato com o autor.

2. Conteúdo geral A. O Web site disponibiliza toda a informação relatada dentro de meus objetivos especificados previamente.

B. As informações estão claramente indicadas e organizadas a fim de serem facilmente entendidas pelos usuários.

C. O conteúdo das informações apresentadas nos links externos é apropriado para a audiência ou usuários.

D. O conteúdo das informações dos Web sites referidos na seção Referências e Fontes Consultadas acrescenta valor à informação.

3. Apresentação A. O design gráfico das páginas favorece o aprendizado.

B. As gravuras ou imagens usadas no Web site agregam conhecimentos aos textos.

C. As gravuras condizem com os textos a elas relacionados.

D. O usuário tem facilidade de navegação página a página, seção a seção, ou de um link para outro, sem ficar confuso ou perdido.

E. A forma de apresentação dos conteúdos em seções ou capítulos, contribui para o aprendizado ou atenção do usuário.

F. O Web site está organizado de maneira clara e lógica, a fim de facilitar a localização dos assuntos.

4. Confiabilidade A. As informações disponibilizadas são confiáveis e acrescentarão conhecimentos sobre a prevenção da Doença Arterial Coronariana.

B. As informações apresentadas estão atualizadas, isto é, existe evidência de que estão sendo atualizadas.

C. As fontes de pesquisa usadas na elaboração do material do Web site são dignas de crédito.

D. A informação está livre de erros tipográficos e gramaticais.

Em cada critério, o consultor pôde pontuar com notas desde zero [péssimo], um [ruim], dois [regular], três [bom], quatro [muito bom] até cinco [excelente]. Assim, para obter a validação, foi estipulado um Limite de Segurança [LS] de 0.81 do total de pontos possíveis, tanto para a análise conjunta da média de cada item, como para a análise individual de cada uma das avaliações, no caso, o total de pontos por avaliação. Dessa forma, para obter a validação, cada item deveria apresentar um escore médio $>$ ou $=$ a 4.05 pontos. Da mesma forma, para o escore total [Grupo I: LSEt=45; Grupo II: LSEt=64]. A análise final dos resultados da validação compreendeu cinco possibilidades representadas na Tabela 3.

Tabela 3 - Análise dos instrumentos de validação

AVALIAÇÄO

GRUPO I GRUPO II

As informaçỗes nẫo contribuem

0 a $11 \quad 0$ a 16

para que os objetivos sejam alcançados.

$O$ alcance dos objetivos depende de complementaçẫo com material adicional de fora do Web site.

O usuário certamente precisará de umguiamais bem estruturado para alcançaros objetivos estabelecidos. o Web site necessita de um mapa para auxiliar o usuário a encontrar certas informaçốes.

O Web site está bem elaborado e $\quad 45$ a $55 \quad 65$ a 80 atinge os objetivos propostos.

\section{RESULTADOS E DISCUSSÃO}

Devido à temática da DAC, o título colocado no Web site foi CardioSite; "Cardio", referindo-se à palavra "coração" e "Site", da palavra "Web site". A construção do CardioSite resultou em um hiperdocumento composto por sessenta e uma páginas, sendo três para a página inicial [ver Figura 1], quarenta e três distribuídas em sete seções do conteúdo temático, nove para a apresentação dos critérios de qualidade, instruções de uso, formulários para registro de opinião e esclarecimento de possíveis dúvidas, mapa do site e seis para o processo de validação. 
As seções do conteúdo temático abordaram a anatomia e fisiologia cardíacas, o processo patológico da DAC, os fatores de risco, os métodos diagnósticos, os tratamentos correntes e as sugestões para a prevenção da doença.

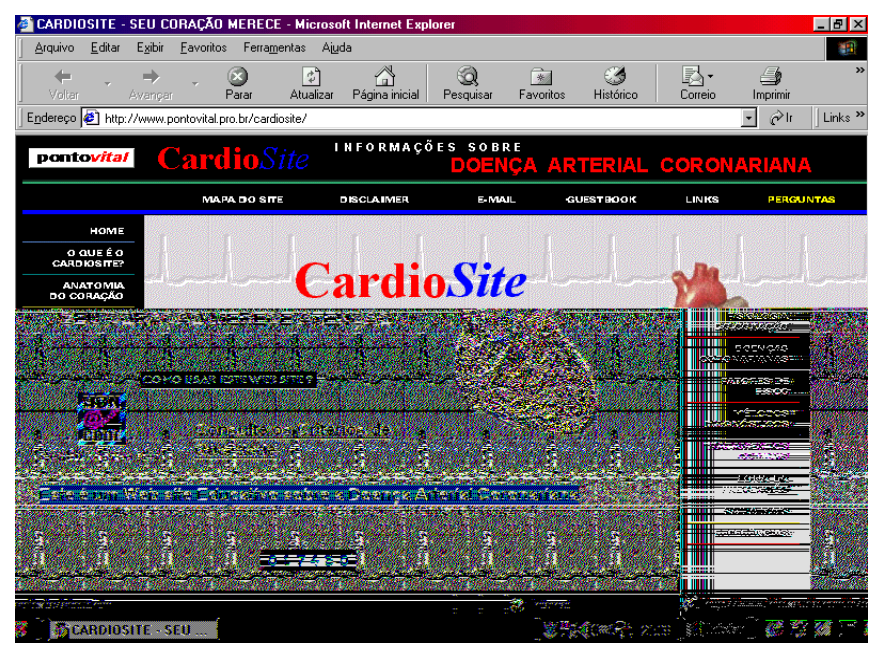

Figura 1 - Home page do CardioSite

A fase de desenvolvimento

A atividade de criação pelo método de desenvolvimento visual demonstrou ser de fácil execução, pois o que era produzido na área de trabalho do software tinha imediata visualização, sem a necessidade de simulação em ambiente do navegador. Por meio desse método, a atividade de desenvolvimento foi mais rápida e efetiva, se comparada com 0 método de programação por códigos HTML.

A forma de apresentação de um determinado conteúdo em página Web é um assunto em constante discussão. Diversos fatores estão envolvidos no complexo processo que ocorre quando uma pessoa acessa a informação de saúde na Web. A maioria dos usuários não lêem todo o conteúdo de uma página Web. O fenômeno que acontece diante da tela do monitor é o escaneamento da informação que interessa à pessoa que a acessa $^{(15)}$. Ao avaliar a qualidade da informação de saúde sob a perspectiva do usuário, é importante considerar que páginas longas e que precisam de barra de rolagem lateral, não são aconselháveis quando se pretende obter uma boa freqüência ao Web site ${ }^{(16)}$. Entretanto, ao considerar o caráter educativo do CardioSite, optou-se pela apresentação do conteúdo em forma de seções com navegação página a página, a partir de ligações (hyperlinks) no seu rodapé, algo que induz o usuário a usar a barra de rolagem e a escanear o conteúdo das páginas mais longas ${ }^{(17)}$.

Critérios de qualidade

Como um dos objetivos do estudo era a obtenção da qualidade no procedimento de validação, durante o desenvolvimento do CardioSite, algumas páginas foram criadas com esse fim. Nessas páginas, foi apresentado o que era o trabalho, quais instituições o apoiavam e as credenciais do autor. Além dessas, foi criada uma página onde foram apresentados os Códigos de Conduta da Fundação $\mathrm{HON}^{(12)}$.

Apesar de que o projeto gráfico e a legibilidade dos materiais usados para a comunicação do conteúdo são importantes para garantir boa freqüência de acesso, esses atributos não causam impacto na qualidade da informação per se ${ }^{(18-19)}$. A autoridade é o primeiro critério ao se avaliar a qualidade de um Web site ${ }^{(12,19)}$. Esse critério diz respeito às credenciais do autor e quais instituições apóiam o trabalho. Outro critério é a confiabilidade das informações. Esse é o ponto mais crítico na avaliação da qualidade de um Web site de saúde. Diversas metodologias já foram sugeridas e descritas, entre elas, a criação de um programa para filtragem e seleção da 
informação ${ }^{(19)}$ e a submissão do material publicado a um Web site chamado SiteLegend ${ }^{(20)}$, o qual avalia todo o material através de sua URL, procurando por doze itens considerados como essenciais para apontar a sua qualidade. Outro fator restritivo ao seu uso é que essas metodologias são programas desenvolvidos para a língua inglesa, algo que limita o seu uso em nosso meio. Outro problema encontrado é que essas ferramentas ainda não atingiram seu propósito definitivo, pois a avaliação da qualidade não está livre de interpretações subjetivas de quem elabora tais programas; algo que pode interferir consideravelmente na aplicação de uma determinada informação, já que sua procura na Web, na maioria das vezes, é baseada numa necessidade ou situação atual que diretamente influencia na tomada de uma decisão ${ }^{(19)}$. Diante desse impasse, na elaboração do desenho do CardioSite, pensou-se na avaliação do conteúdo sob a perspectiva do profissional de saúde e de informática como uma forma de validá-lo, garantindo, assim, a sua qualidade.

\section{A validação}

O processo de validação do qual participaram os consultores do Grupo I [n=6], apresentou a média do escore total de 48.6 \pm 2.9 pontos e, a média do escore por itens de $4,42 \pm 0.34$ pontos. Na avaliação discriminada por itens [ver Figura 2], dois deles obtiveram a média inferior ao LSEi [4.05 pontos]. Estes itens estavam relacionados ao funcionamento eficiente dos hyperlinks para páginas de Web sites externos (critério 4 , item $\mathrm{C}=$ média 3,8 pontos) e à qualidade da apresentação gráfica de algumas imagens (critério 5 , item $A=$ média 3,8 pontos). O resultado final do processo de validação com este grupo caracterizou 0
CardioSite como bem elaborado e que atingiu os objetivos propostos.

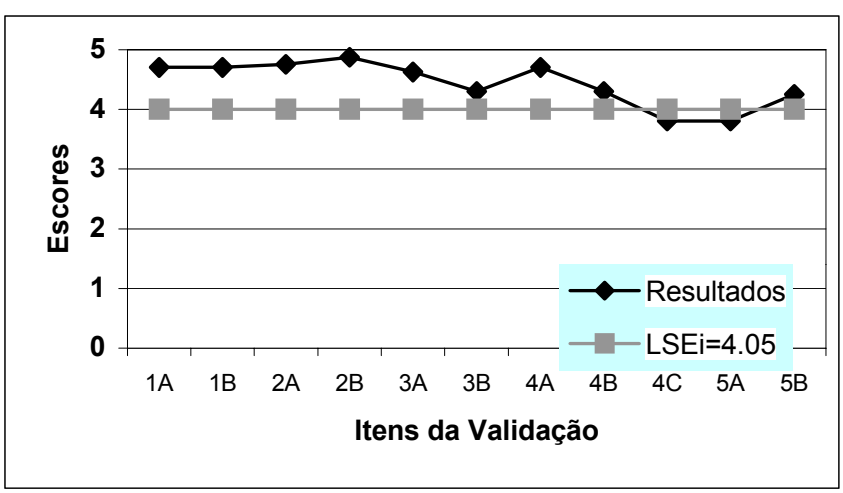

Figura 2 - Avaliação por itens feita pelos profissionais de informática (Grupo I)

Com o Grupo II [n=11], a validação apresentou a média de $72.2 \pm 1.8$ pontos e a média do escore por itens de $4.51 \pm 0.28$ pontos. $\mathrm{Na}$ avaliação discriminada por itens [ver Figura 3], apenas um deles apresentou média inferior ao LSEi [4,05 pontos]. Esse item estava relacionado à presença de erros tipográficos $\mathrm{e}$ gramaticais [critério 4 , item $\mathrm{D}=$ média 3,7 pontos]. Como resultado final, o processo de validação com esse grupo também caracterizou o CardioSite como bem elaborado e que atingiu os objetivos propostos. Analisando os itens em que se obteve pontuação inferior ao LSEi, procedeu-se a revisão dos hiperlinks externos, a re-edição de algumas imagens, e a correção dos erros tipográficos e gramaticais conforme a indicação dos consultores.

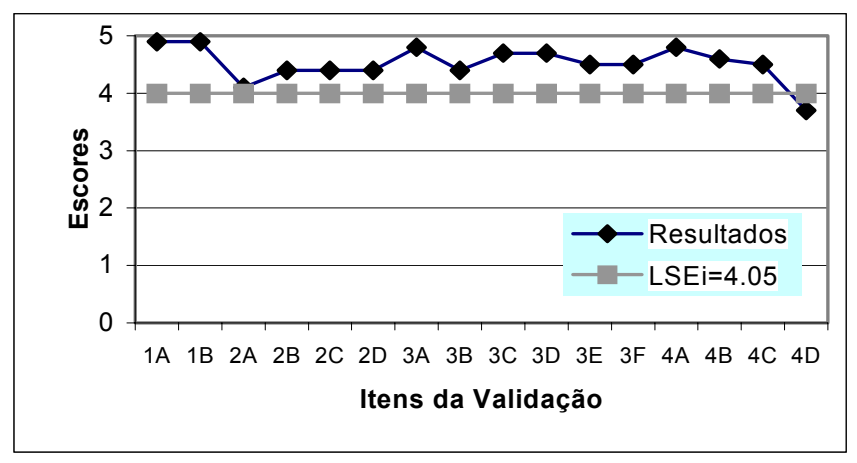

Figura 3 - Avaliação por itens feita pelos profissionais da área de saúde (Grupo II) 
O processo de validação, ao qual foi submetido o CardioSite, foi um elemento adicional para avaliar a qualidade da informação produzida. Sob a óptica da literatura ${ }^{(13,16)}$, esse processo pode se tornar redundante, caso os Códigos de Conduta da Fundação $\mathrm{HON}^{(12)}$ já tenham sido previamente adotados. Todavia, como em nosso país inexistem métodos ou sistemas oficiais que controlem ou revisem a qualidade da informação de saúde publicada na $W e b$, o painel on-line com especialistas serviu para confirmar a qualidade do CardioSite.

Completando o processo de implementação, o CardioSite foi cadastrado nas principais bases de dados das ferramentas de busca nacionais e de outros países de língua portuguesa, liberando a sua utilização para o usuário alvo.

\section{CONCLUSÕES}

A partir dos resultados descritos e das observações realizadas durante a elaboração do CardioSite, em suas fases de modelagem conceitual, de desenvolvimento e de implementação, conclui-se que: a primeira fase foi a que empreendeu o maior gasto de tempo, por exigir extensa pesquisa bibliográfica para a seleção e elaboração de seu conteúdo. Nesse

\section{REFERÊNCIAS BIBLIOGRÁFICAS}

1. Rosa CAS. Internet: história, conceitos e serviços. São Paulo (SP): Editora Érica; 1998.

2. Edwards MJA. The Internet for nurses and allied health professionals. New York (NY): Springer-Verlag; 1995.

3. Marin HF. Nursing informatics in Brazil: a brazilian experience. Comp Nurs 1998 Nov-Dec; 16(6):327-32.

4. Simpson RL. Will the Internet supplant community health networks? Nurs Manag 1996 February; 27 (2): 20-3. processo, a adoção dos modelos de Web sites da American Heart Association e InteliHealth foi de grande utilidade. Para atribuir qualidade à informação, os Códigos de Conduta da Fundação $\mathrm{HON}^{(12)}$ e as diretrizes do Health Information Technology Institute ${ }^{(14)}$ foram importantes para o preparo do material sob essa perspectiva, garantindo que a validação do fosse alcançada com êxito.

Durante a fase de desenvolvimento, pode-se dizer que o trabalho de criação com o método de desenvolvimento visual foi uma atividade fácil e que exigiu conhecimentos básicos de microinformática. A partir dessa conclusão, é possível afirmar que a atividade pode ser executada facilmente por pessoas que detém conhecimentos básicos de microinformática. Nesse caso, enfermeiros interessados podem ser capazes de desenvolver suas próprias páginas Web, independente da finalidade para a qual elas se destinam.

Quanto à fase de avaliação, o processo de validação por meio do painel on-line com especialistas das áreas de informática e saúde, foi efetivo para conferir qualidade às informações contidas no CardioSite e que, em face da inexistência de sistemas nacionais oficiais com esse propósito, pode-se dizer que a elaboração desse modelo foi suficiente para atingir o objetivo estabelecido.

5. Miller PA, Carlton $\mathrm{KH}$. Technology as a tool for health care collaboration. Comp Nurs 1998 Jan-Feb; 16 (1): 27-9.

6. Klenn P, Reppert K, Visich L. A nontraditional cancer support group: the Internet. Comp Nurs 1998 JanFeb;16(1):31-6.

7. Bliss J, Alibone C, Bontempo B, Flynn T, Valvano NE. Creating a Web site for on-line social support: melanocyte. Comp Nurs1998 Jul-Aug;16(4):203-7.

8. Sparks SM. Using the Internet for urology nursing. Urol Nurs 1996 December;16(4):131-4. 
9. Gold J. Mental health and the Internet. Comp Nurs 1998 Mar-April;16(2):85-6.

10. Silva FB da, Cassiani SH de B, Zem-Mascarenhas $\mathrm{SH}$. A internet e a enfermagem: construção de um site sobre administração de medicamentos. Rev Latino-am enfermagem 2001 janeiro;9(1):116-22.

11. Ministério da Saúde (BR). Doenças cardiovasculares no Brasil: dados estatísticos. [citado em 199910 fev.] Disponível em: URL: http://www.saude.gov.br

12. Trochim WMK. Evaluating Web sites. Cornell University, 1996. [citado em 199910 maio]. Disponível em: URL: http://trochim.human.cornell.edu/webeval/ webintro.htm

13. Health On the Net Foundation. HON Code of conduct for medical and health related Web sites. Versão 1.6, 1997. [citado em 199925 out.]. Disponível em: URL: http:/ /www.hon.ch

14. Health Information Technology Institute. Criteria for assessing the quality of health information on the Internet. 1997. [citado em 200012 jul.]. Disponível em: URL: http:/ /www.mitrek.org/hiti/showcase/documents/criteria.html

15. Joseph L. WWW cyberguide ratings for content evaluation. Cyberguides, 1999. [citado em 199925 out.]. Disponível em: URL: http://www.cyberbee.com/ guide1.html

16. Morkes J, Nielsen J. Concise, scannable, and objective: how to write for the Web. Useit.com, 1997. [citado em 200014 maio]. Disponível em: URL: http:// www.useit.com/papers/webwriting.html

17. Jones J. Development of self-assessment method for patients to evaluate health information on the Internet. Proc AMIA Symp [serial online] 1999:540-4. Available from: URL: http://www.amia.org/pubs/symposia/ D005629.PDF

18. Knox ELS. The pedagogy of Web site design. ALN Magazine [serial online] 1997 ago; 1(2): [10 screens]. Available from: URL:http://www.aln.org/alnweb/issue2/ knox.htm

19. Jadad A, Gagliardi A. Rating health information on the Internet. JAMA 1998 February; 278(8):611-4.

20. Eysenbach G, Diepgen TL. Towards quality management of medical information on the Internet: evaluation, labelling, and filtering of information. Br Med J 1998 November; 317(7171):1496-502.

21. Weiler RM, Pealer LN. The SiteLegend: twelve components of a new strategy for providing website documentation. J Sch Health 2000 April;70(4):148-52. 